\title{
Flow optimization of electrostatic precipitator using non uniform porosity distribution screen
}

\author{
Sunil J. Kabadi* and Manoj D. Hambarde \\ ${ }^{\dagger}$ Mechanical Department, Pune University, MIT, kothrud, pune, India \\ Accepted 15 June 2016, Available online 20 June 2016, Special Issue-5 (June 2016)
}

\begin{abstract}
Electrostatic precipitator (ESP) require uniform flow distribution to achieve better collection efficiency. Perforated plates are widely used as distributing member in fluid flow control. For better flow distribution three perforated plates are used. Flow pattern inside the collection zone is strongly affected by diffuser angle, porosity, and location within the diffuser. The present investigation aims at simulation of flow behaviour within electrostatic precipitator and finding standard porosity pattern for various ESP's for avoiding flow separation and achieving high degree of flow uniformity. Computational fluid dynamics (CFD) modelling of gas flow is becoming a valuable tool in designing, testing, and comparing different solutions. In present study flow pattern of 23 geometries of ESP's are simulated and they are divided in to six groups according to inlet opening ratio and standard porosity pattern is find out for each group .
\end{abstract}

Keywords: electrostatic precipitator, computational fluid dynamics, flow pattern, porosity.

\section{Introduction}

Electrostatic precipitator (ESP) is widely used in industries as emission control unit. For achieving better collection efficiency, the flow inside the electrostatic precipitator should be slow moving and uniformly distributed. Wide angle diffuser at inlet reduces the velocity up to one tenth of inlet velocity. When flow enters the diffuser at inlet it faces an adverse pressure gradient that result in flow separation which causes flow concentration in localised area and non-uniform flow distribution. The conventional solution to this problem is to use the perforated screens for controlling flow at upstream of treatment zone. For controlling the flow three gas distribution screens are used. It is observed that uniform porosity distribution screens are unable to distribute the flow uniformly because along screen high velocity as well as low velocity regions are present. By keeping low percentage opening at high velocity region and high percentage opening at low velocity region the flow can be distributed uniformly so non uniform porosity distribution screen is one of the best solution.

Generally pyramidal wide angle diffuser is used to expand the flow, but because of wide angle and short length of diffuser flow experience an adverse pressure gradient. In absence of internal flow control, the flow

*Corresponding author: Sunil J. Kabadi separates from the diffuser walls very close to the point where they start to diverge. Because of wide angle large regions of reversed flow are formed on the wall of diffuser. Central high velocity flow continues travel straight in the diffuser and form axial high velocity region at diffuser exit. The sideway flapping motion may also be establish.

\section{Mesh generation}

The present study is based on application of computational fluid dynamics (CFD) to the electrostatic precipitator (ESP). Modeling of fluid flow requires the specification of the geometry through a computational grid. The geometry chosen for simulation properly represent the physics of problem.

\section{1 modelling}

Auto Cad drawing received from customer is studied well and as per customer specifications 3D Geometry is drown and mesh using Ansys ICEM. Structured hexahedral grids is used for meshing. Ansys ICEM is a popular proprietary software package used for CAD and mesh generation. Model already drown in to other CAD packages can be imported in to ICEM for meshing purpose. Fig. 1 shows 3 module ESP drown by using Ansys ICEM. 


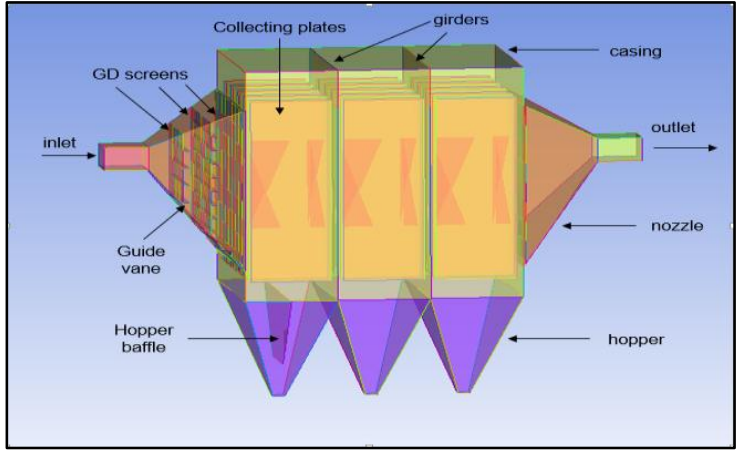

Fig.1 Typical 3 module ESP geometry

\section{2 meshing}

In meshing, domain is discretized in to a finite set of control volumes or cells. Discretized domain is called as grid or mesh. Unstructured grid generation is usually much faster than structured grid generation. For simple and less complicated geometries, structured grid are more accurate. Structured grid calculations usually takes less time than an unstructured grid calculation because, to date, the existing algorithms are more efficient. For generating structured mesh blocking method is used. In blocking appropriate blocks are created for each part of the geometry and for capturing the internal surfaces association with the blocking is done. Fig. 2 shows blocking and association for geometry and fig.3 shows computational hexahedral mesh.

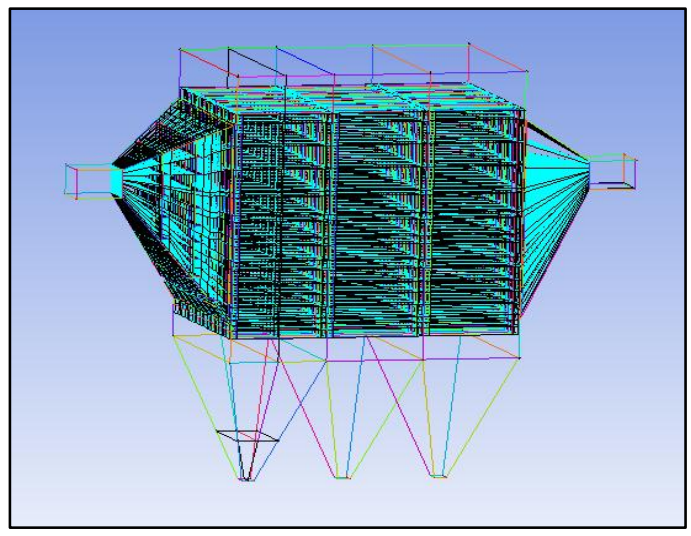

Fig.2 blocking

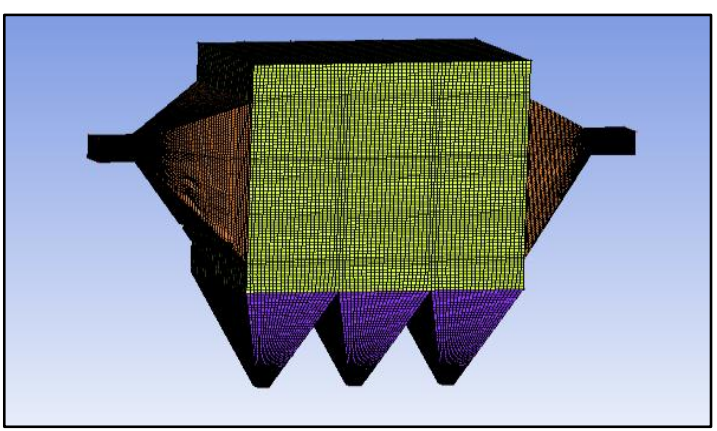

Fig.3 computational mesh of ESP
Modelled computational mesh that represent full flow simulation model includes all details that can affect the gas flow filled. This includes gad distribution screens, guide vanes, hopper baffle, casing, collecting plates and other flow obstructions. Computational meshes varies from $11,00,000$ to $25,00,000$ depending on the size of ESP and accuracy needed. Large number computational cells results in very high resolution of flow field.

\section{Model Simulation}

Mesh file created by ICEM is imported in to Ansys Fluent. Turbulence is modelled by the standard K- $\varepsilon$ model which is widely used for industrial internal flows.

\subsection{Governing equations}

For all flows, FLUENT solves numerically the Conservation Equations for Mass, Momentum and additional Transport equations (if the flow is turbulent) for a fluid in a given flow geometry. The transport equations have different forms depending on the turbulence modeling used. Bases for the CFD analysis of the flow inside the ESP has been set as realizable $\mathrm{k}-\varepsilon$ turbulence model, steady state incompressible flow; and hence, all the governing equations are based upon those physical flow models.

For flows general equation for conservation of mass or continuity equation can be written as follows:

$\frac{\partial \rho}{\partial t}+\operatorname{div}(\rho u)=0$

Navier-Stokes Equations

$\frac{\partial(\rho u)}{\partial t}+\operatorname{div}(\rho u u)=-\frac{\partial P}{\partial X}+\operatorname{div}(\mu \operatorname{grad} u)+$

$\left[-\frac{\partial\left(\rho \overline{u^{\prime 2}}\right)}{\partial X}-\frac{\partial\left(\rho \overline{u^{\prime} v^{\prime}}\right)}{\partial Y}-\frac{\partial\left(\rho \overline{u^{\prime} w^{\prime}}\right)}{\partial Z}\right]+S_{\Phi x}$

$\frac{\partial(\rho v)}{\partial t}+\operatorname{div}(\rho v u)=-\frac{\partial P}{\partial Y}+\operatorname{div}(\mu \operatorname{grad} v)+$

$\left[-\frac{\partial\left(\rho \overline{u^{\prime} v^{\prime}}\right)}{\partial X}-\frac{\partial\left(\rho \overline{v^{\prime 2}}\right)}{\partial Y}-\frac{\partial\left(\rho \overline{v^{\prime} w^{\prime}}\right)}{\partial Z}\right]+S_{\Phi y}$

$\frac{\partial(\rho w)}{\partial t}+\operatorname{div}(\rho w u)=-\frac{\partial P}{\partial z}+\operatorname{div}(\mu \operatorname{grad} w)+$

$\left[-\frac{\partial\left(\rho \overline{u^{\prime} w^{\prime}}\right)}{\partial X}-\frac{\partial\left(\rho \overline{v^{\prime} w^{\prime}}\right)}{\partial Y}-\frac{\partial\left(\rho \overline{w^{\prime 2}}\right)}{\partial Z}\right]+S_{\Phi Z}$

Two equation model ( $\mathrm{k}-\varepsilon$ model)

In two-dimensional thin shear layers the changes in the flow direction are always so slow that the turbulence can adjust itself to local conditions. If the convection and diffusion of turbulence properties can be neglected it is possible to express the influence of turbulence on the mean flow in terms of the mixing length. If convection and diffusion are not negligible-as is the 
case for example in recirculating flows-a compact algebraic prescription for the mixing length is no longer feasible. The mixing length model lacks this kind of generality. The way forward is to consider statements regarding the dynamics of turbulence. The $\mathrm{k}-\varepsilon$ model focuses on the mechanisms that affect the turbulent kinetic energy. Some preliminary definitions are required first. The instantaneous kinetic energy $\mathrm{K}$ (t) of a turbulent flow is the sum of the mean kinetic energy and the turbulent kinetic energy.

$\mathrm{K}(\mathrm{t})=\mathrm{K}+\mathrm{k}$,

$K=\frac{1}{2}\left(\bar{u}^{2}+\bar{v}^{2}+\bar{w}^{2}\right)$

$k=0.5\left(\overline{u^{\prime 2}}+\overline{v^{\prime 2}}+\overline{w^{\prime 2}}\right)$

Transport Equations for the k- $\varepsilon$ Model

The turbulence kinetic energy, $k$, and its rate of dissipation, $\varepsilon$, are obtained from the following transport equations

$\frac{\partial}{\partial \mathrm{t}}(\rho \kappa)+\frac{\partial}{\partial \mathrm{x}_{\mathrm{I}}}\left(\rho \kappa \mathrm{i}_{\mathrm{i}}\right)=\frac{\partial}{\partial \mathrm{x}_{\mathrm{j}}}\left[\left(\mu+\frac{\mu_{\mathrm{t}}}{\sigma_{\kappa}}\right) \frac{\partial \kappa}{\partial \mathrm{x}_{\mathrm{j}}}\right]+\mathrm{G}_{\mathrm{k}}+\mathrm{G}_{\mathrm{b}}-$

$\rho \epsilon-\mathrm{Y}_{\mathrm{M}}+\mathrm{S}_{\mathrm{\kappa}}$

$\frac{\partial}{\partial \mathrm{t}}(\rho \varepsilon)+\frac{\partial}{\partial \mathrm{x}_{\mathrm{j}}}\left(\rho \varepsilon \mathrm{u}_{\mathrm{j}}\right)=\frac{\partial}{\partial \mathrm{x}_{\mathrm{j}}}\left[\left(\mu+\frac{\mu_{\mathrm{t}}}{\sigma_{\varepsilon}}\right) \frac{\partial \varepsilon}{\partial \mathrm{x}_{\mathrm{j}}}\right]+\rho \mathrm{C}_{1} \mathrm{~S}_{\varepsilon}-$

$\rho \mathrm{C}_{2} \frac{\varepsilon^{2}}{\kappa+\sqrt{v \varepsilon}}+\mathrm{C}_{1 \varepsilon} \frac{\varepsilon}{\kappa} \mathrm{C}_{3 \varepsilon} \mathrm{G}_{\mathrm{b}}+\mathrm{S}_{\varepsilon}$

Where,

$C_{1}=\max \left[0.43, \frac{\eta}{\eta+5}\right], \eta=S \frac{\kappa}{\varepsilon}, S=\sqrt{2 S_{i j} S_{i j}}$

In these equations, $\mathrm{G}_{\mathrm{k}}$ represents the generation of turbulence kinetic energy due to the mean velocity gradients, $G_{b}$ is the generation of turbulence kinetic energy due to buoyancy, YM represents the contribution of the fluctuating dilatation in compressible turbulence to the overall dissipation rate, $\mathrm{C}_{1} \& \mathrm{C}_{2}$ are constants. $\Sigma_{\mathrm{k}}$ and $\sigma_{\varepsilon}$ are the turbulent Prandt numbers for $\mathrm{k}$ and $\varepsilon$, respectively. $S_{\mathrm{k}}$ and $S_{\varepsilon}$ are userdefined source terms. The model constants $C_{1 \varepsilon}, C_{2}, \sigma_{\varepsilon}$ and $\sigma \varepsilon$ have the following default values: $\mathrm{C}_{1 \varepsilon}=1.44, \mathrm{C}_{2}=$ $1.9, \sigma_{\varepsilon}=1.0, \sigma_{\varepsilon}=1.2$

\subsection{Model approximations}

Main purpose of The CFD is to analyze the gas flow distribution and pressure loss. The effect of particle concentration and distribution in the ESP is not considered in the simulation.

Flow is considered as time independent (steady state), incompressible and isothermal with fluid properties at given operating temperature.
1) Atmospheric air is considered as working fluid.

2) Analysis is done for steady state conditions

3) Analysis is done for single phase

4) A uniform velocity profile is assumed at the inlet of the computational mesh.

5) Porosity values for perforated sheet openings are considered as per the company standard.

\subsection{Boundary condition}

Boundary condition is stated restriction, usually in the form of an equation that limits the possible solutions to differential equation.

Inlet - velocity inlet.

Outlet - pressure outlet.

Gas distribution screen - porous jump.

Nozzle, casing, hopper, hoper baffle, girders, collecting plates - wall.

Flow rate of all ESP is given in Auto CAD sheet and inlet area can be calculated by using inlet duct dimensions. By dividing the mass flow rate by inlet area inlet velocity can be calculated. As inlet velocity is known, velocity boundary condition can be assigned to inlet. Pressure at an outlet of ESP is atmospheric pressure so pressure boundary condition can be assign to outlet. Gas distribution screens are perforate plates with circular holes with very small diameter. It is very difficult to capture the thousands of circular holes of very small diameter and create mesh along the curve portion of hole. By doing this mesh count goes very high and calculation time increase tremendously. To avoid high mesh count and for saving time, porous media boundary condition is used for GD screen.

\section{Gas Distribution screen modelling}

Generally three gas distribution screens are used for distributing the gas flow uniformly in the diffuser.

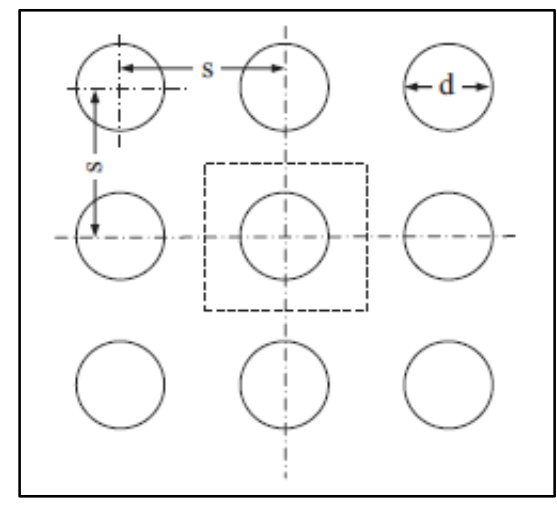

Fig.4 Representative Unit cell 
The thickness of GD screen is very small and number of small diameter holes are punched on the screen. Diameter of hole is constant so, porosity is varied by varying the pitch of the holes. As pitch is increased the distance between the holes is increased and percentage open area decreased and vice versa. There are different openings used for GD screens such as 23\%, $30 \%, 40 \%$, and $50 \%$.

It is very difficult to control the flow with uniform porosity distribution screens because of adverse pressure gradient and wall attached flow there is uneven flow concentration along the screen.

Porous media of finite thickness with permeability over which the pressure drop is defined is a combination of Darcy's Law and an additional inertial loss term is given by

$\Delta \mathrm{p}=\left\{\frac{\mu}{\alpha} \vartheta+\mathrm{C}_{2} \frac{1}{3} \rho \vartheta^{2}\right\} \Delta \mathrm{m}$

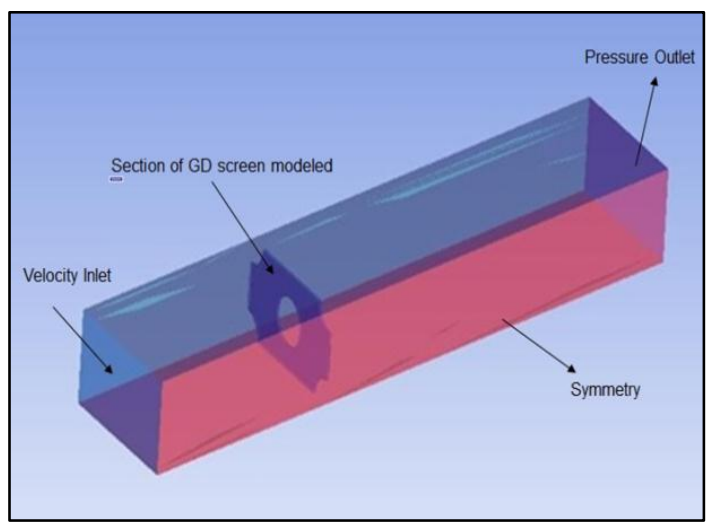

Fig5. Selection of unit cell from GD screen.

Porous jump coefficient $\mathrm{C}_{2}$ and face permeability $\alpha$ of GD screen are generally calculated from experimental and mathematical modelling. For calculating values of Porous jump coefficient $\left(\mathrm{C}_{2}\right)$ and face permeability $(\alpha)$ simulation of unit cell with fluid flow domain is done. For each percentage opening, separate unit cell of GD screen is modelled and simulation is carried for different velocity to calculate the pressure drop across inlet and outlet which is occurred due to inertial loss at turbulent flow. Fig 4 shows a modelled and mesh file of unit cell of a $30 \%$ opened GD screen.

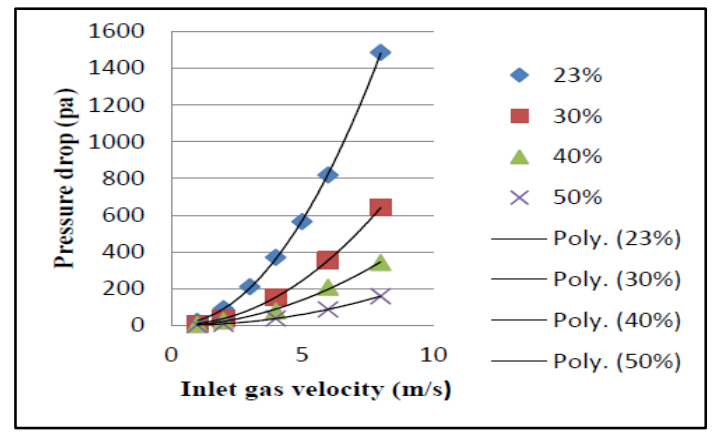

Fig.6 Relations between inlet gas velocity and pressure drop for different GD percentage opening.
Same simulation is repeated for opening of $23 \%, 30 \%$, $40 \%$, and $50 \%$. The corresponding parameters such as porous jump coefficient $\mathrm{C}_{2}$ and face permeability $\alpha$ are obtained by curve fitting given in Table 1 . A second degree polynomial curve is obtained for every opening and it is solved for equation (8). Fig 6 shows that pressure drop increased with increase in inlet velocity and reduction in porosity.

Table 1 Porous jump Coefficient and Face Permeability values for different percentages of GD openings.

\begin{tabular}{|c|c|c|c|}
\hline $\begin{array}{c}\text { Sr. } \\
\text { No. }\end{array}$ & $\begin{array}{c}\text { GD \% } \\
\text { opening }\end{array}$ & $\begin{array}{c}\text { Face } \\
\text { permeability } \\
\boldsymbol{\alpha}\left(\mathrm{m}^{2}\right)\end{array}$ & $\begin{array}{c}\text { Porous } \\
\text { jump } \\
\text { coefficient } \\
\mathrm{C}_{2}\left(\mathrm{~m}^{-1}\right)\end{array}$ \\
\hline 1 & 23 & $2.10 \mathrm{E}-08$ & 12763 \\
\hline 2 & 30 & $2.13668 \mathrm{E}-08$ & 5614 \\
\hline 3 & 40 & $7.36177 \mathrm{E}-08$ & 2896 \\
\hline 4 & 50 & $9.35389 \mathrm{E}-08$ & 1360 \\
\hline
\end{tabular}

\section{Acceptance criteria}

\subsection{Velocity readings}

All the ESP's should follow the ICAC guidelines (3) for uniform flow distribution for attaining maximum efficiency. The velocity pattern shall have a minimum of $85 \%$ of the velocities not more than 1.15 times the average velocity and $99 \%$ of the velocities not more than 1.40 times the average velocity. Average velocity refers to the mean of all velocity measurements made at a given face of the precipitator. As per ICAC guidelines (3) all this velocities should be measured near the inlet and outlet faces of the precipitator collection chamber, where as we measured at the end of first field. Table 4 shows the readings of velocity at end of first field of ESP.

Table 2 Velocity measurement sheet

\begin{tabular}{|c|r|r|r|r|r|r|}
\hline 1 & \multicolumn{1}{|c|}{2} & 3 & \multicolumn{1}{c|}{4} & \multicolumn{1}{c|}{5} & \multicolumn{1}{c|}{6} & \multicolumn{1}{c|}{7} \\
\hline 0.527211 & 0.44659 & 0.436138 & 0.465104 & 0.46315 & 0.413165 & 0.391193 \\
\hline 0.554631 & 0.48548 & 0.465318 & 0.448079 & 0.393677 & 0.319345 & 0.429365 \\
\hline 0.444345 & 0.359789 & 0.332788 & 0.322961 & 0.27236 & 0.330373 & 0.443482 \\
\hline 0.371016 & 0.263762 & 0.282983 & 0.332439 & 0.40759 & 0.467096 & 0.403928 \\
\hline 0.404758 & 0.353873 & 0.443024 & 0.510903 & 0.521449 & 0.406567 & 0.468283 \\
\hline 0.506377 & 0.528177 & 0.534034 & 0.460907 & 0.395032 & 0.501979 & 0.778003 \\
0.621159 & 0.70897 & 0.749271 & 0.680641 & 0.795291 & 0.933625 & 1.00389 \\
0.787115 & 0.920957 & 0.959602 & 0.97589 & 0.983698 & 0.963136 & 0.932959 \\
\hline
\end{tabular}

Table 3 Velocity criteria check sheet

\begin{tabular}{|c|c|c|c|}
\hline Average velocity (V avg.) & 1.09 & $\mathrm{~m} / \mathrm{sec}$ & \\
\hline 1.15 times V avg. & 1.25 & $\mathrm{~m} / \mathrm{sec}$ & \\
\hline 1.40 times V avg. & 1.52 & $\mathrm{~m} / \mathrm{sec}$ & \\
\hline Total Velocity Readings & 180 & Nos. & \\
\hline $\begin{array}{c}\text { No. of readings within } 1.15 \\
\text { times V avg. }\end{array}$ & 156 & In \% & $87 \%$ \\
\hline $\begin{array}{c}\text { No. of readings within } 1.40 \\
\text { times V avg. }\end{array}$ & 179 & In \% & $99 \%$ \\
\hline Standard Deviation & 0.16 & & \\
\hline RMS in \% & $14 \%$ & In \% & \\
\hline
\end{tabular}




\subsection{RMS value}

The percent RMS is calculated by the following formula

$\%$ RMS $=\frac{100}{V_{\text {avg }}} \sqrt{\frac{\sum\left(V_{i}-V_{\text {avg }}\right)^{2}}{\left(\sum i\right)-1}}$

Where,

$V_{\mathrm{i}}=$ velocity at selected grid point on the rake

$V_{a v g}=$ average velocity over entire plane

$\mathrm{I}=$ grid point counter

The typical goal in industry is to achieve a Percent RMS of less than $15 \%$ at the ESP inlet and outlet planes where as we measured RMS value at the end of first field.

\section{Discussion and result}

Pyramidal Wide angle diffuser is commonly used in electrostatic precipitator for gas flow distribution. It converts the kinetic energy of flow in to pressure energy by decelerating the flow in the direction of fluid motion. Pyramidal Wide angle diffuser has larger diffusion angle and area ration than common diffuser. When flow enters the diffuser inlet it faces the adverse pressure gradient because of this adverse pressure gradient, flow separation takes place. With presence of flow separation pressure rise capability of diffuser decrease and increase in pressure loss take place. Fig. 6 shows wide angle diffuser

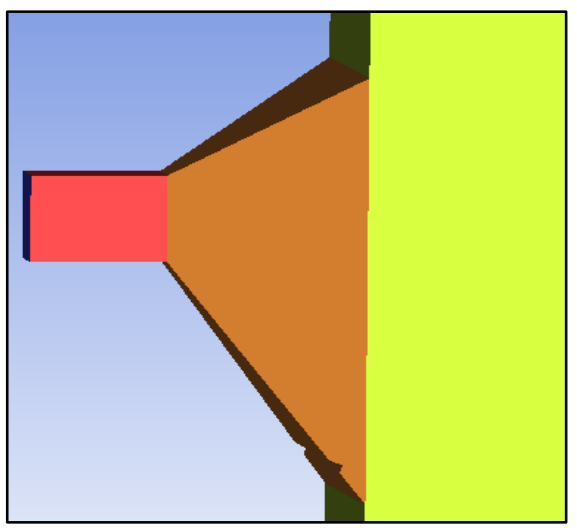

Fig.7 Wide angle diffuser

Without any form of flow control flow emerging from diffuser is unevenly distributed. Thin perforated plates with thickness $3 \mathrm{~mm}$ and hole diameter of $35 \mathrm{~mm}$ are used to control and distribute flow uniformly. Fig.7 shows actual picture of gas distribution screen. Three gas distribution screens are used in the inlet diffuser and one in the outlet diffuser. In inlet diffuser they are placed equidistance with first gas distribution screen at certain distance from the inlet and third close to exit of diffuser.
ESP mesh used for simulation have 14,00,0000 computational cells grid independency test is taken and simulation done on Core i7 $3.1 \mathrm{GHz} 64$ bit CPU with 8 GB RAM and 1TB hard disc memory. FLUENT 14.5 is used for simulation.

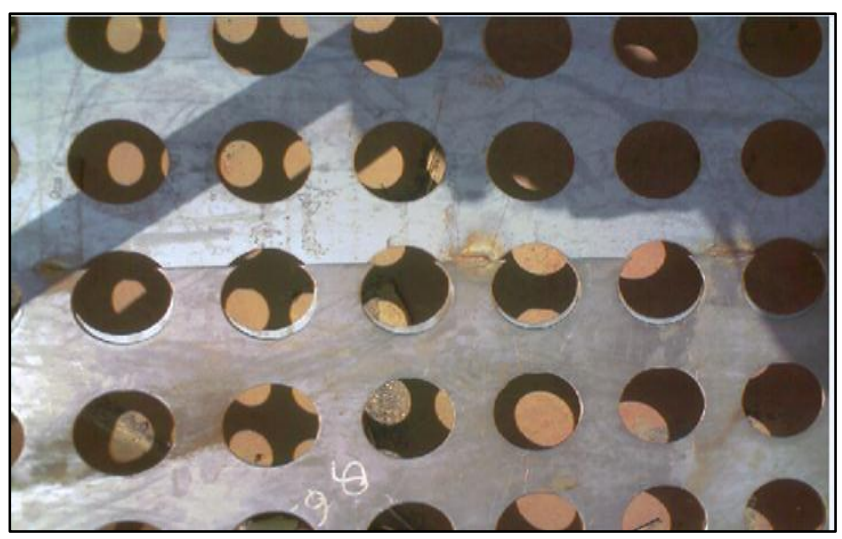

Fig.8 gas distribution screen

As flow enters the diffuser because of gradual change in area ration it continues linear motion and flows linearly normal to axis. When flow comes out from first gas distribution screen it experience adverse pressure gradient because of drastic change in area ration in the direction of flow. As flow travels kinetic energy of flow is converted in to rise in pressure energy by decelerating the flow in fluid motion. After passing first gas distribution screen axisymmetric flow get distributed to wall of diffuser and central low velocity region is created. Recirculation zone is observed after first screen.

When flow strikes the gas distribution screen some dust particle are stick to the screen and after some time they fall down and collected at the bottom of diffuser. For removing this dust $75 \mathrm{~mm}$ height opening is kept at the bottom of all gas distribution screen. Large amount of flow escape through this opening which result in high velocity flow at the bottom. Fig. 9 shows velocity contour at vertical mid-section of ESP.

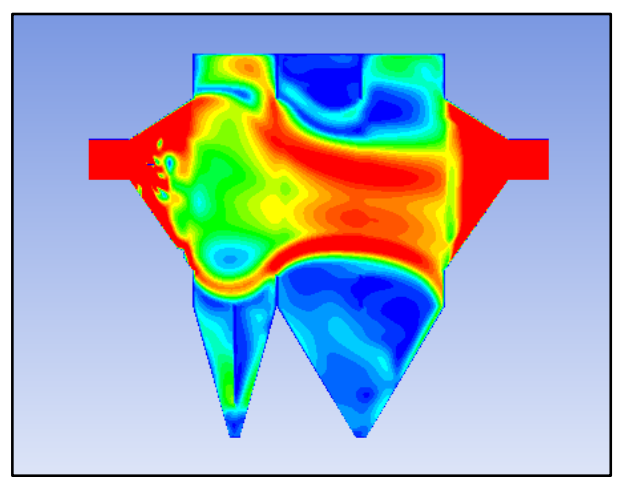

Fig.9 Velocity contour at vertical mid-section of ESP before modification

We can see in fig. 8 maximum flow is flowing from top and bottom of the diffuser and after emerging out from 
diffuser high velocity flow observed from bottom to the middle of collecting plate.

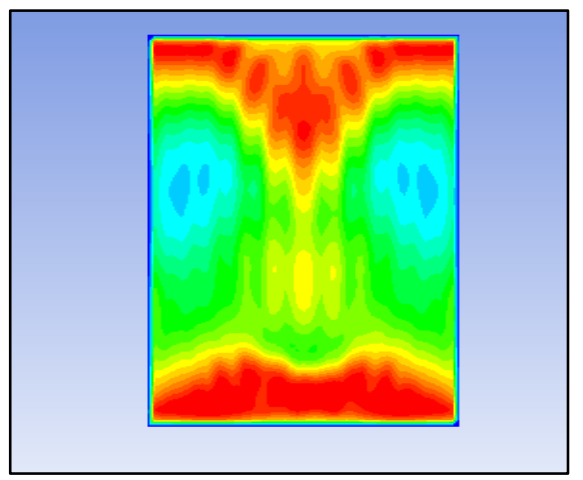

Fig.10 Velocity contour at end of first field of ESP before modification

High velocity flow is observed at the top and bottom of first field and low velocity gaps are observed at right and left hand side. Moderate velocity flow is observed vertically middle of the field. It is clear from fig. 8 that flow is not uniformly distributed and it not follow the ICAC criteria. Percentage opening kept for second inlet gas distribution screen is shown in fig.11 (A)

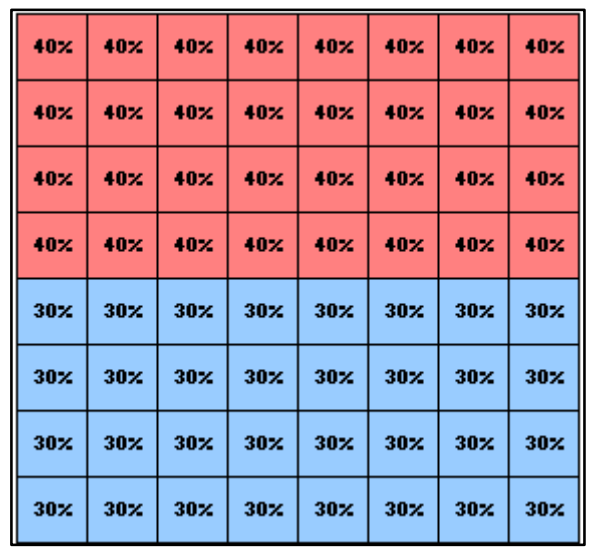

(A)

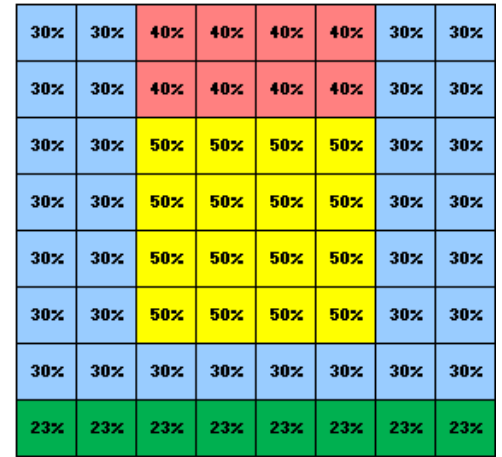

(B)
Fig.11 Second gas distribution screen opening percentage. (A) Before optimization. (B) After optimization.

Velocity profile along the first gas distribution screen is uniform it not need to change. But for gas distribution screen second and third it need to be optimized for achieving uniform flow. Along second gas distribution screen high velocity is observed at left and right hand side of the plate also because of provision kept for collected dust large amount of high velocity flow flows through bottom of screen.

For restricting flow at right and left side of gas distribution screen velocity percentage reduced from $40 \%$ to $30 \%$. For restricting high velocity flow at bottom opening percentage reduced from $30 \%$ to $23 \%$.

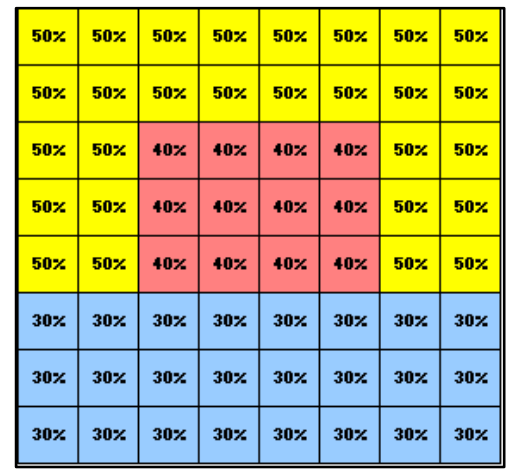

(A)

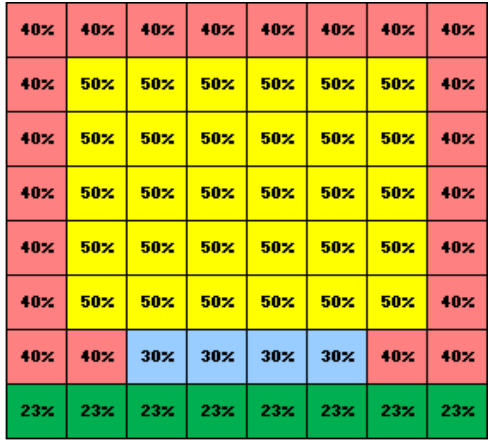

(B)

Fig.12 Third das distribution screen opening percentage. (A) Before optimization. (B) After optimization.

It is observed that because of centrally out ward flow after first gas distribution screen very low velocity patch is occurred at central portion of second gas distribution screen. To allow increase the flow at middle portion, $50 \%$ opening is kept at central portion. Optimized gas distribution screen percentage is shown in fig. 10 (B).

Third gas distribution screen before optimization is shown in fig 12 (A). It is observed that high velocity flow flows along the four sides of the diffuser so less percentage opening is kept at sides of the diffuser and percentage opening reduced to $40 \%$. To restrict high 
velocity flow at the bottom opening of screen very less opening $(23 \%)$ is kept at the bottom of screen shown in fig12 (B).

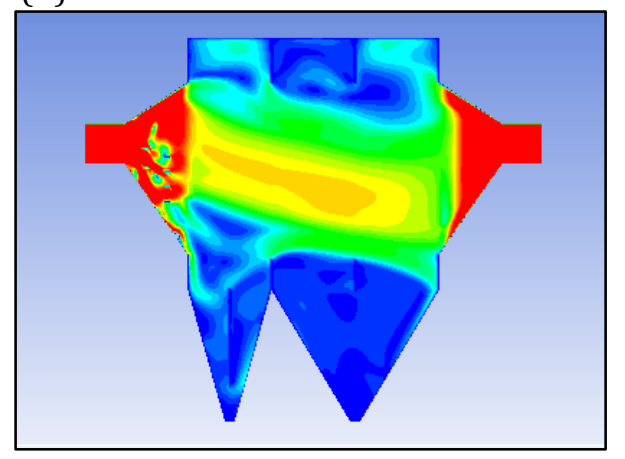

Fig.13 Velocity contour at vertical mid-section of ESP after modification

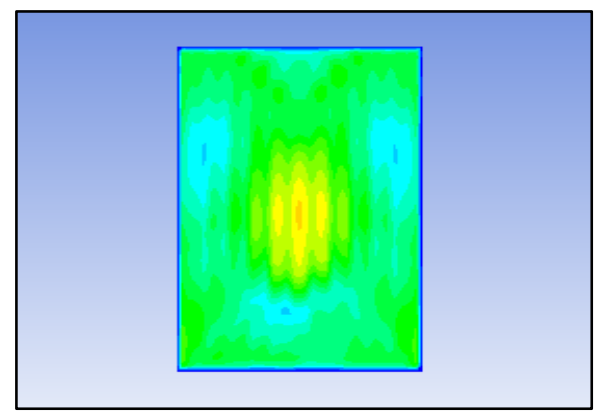

Fig.14 Velocity contour at end of first field of ESP after modification

As we can see from fig.13 that flow is uniformly distributed on mid-section of ESP. high velocity region at the bottom of casing is removed. Fig.14 shows velocity contour at end of first field. High velocity patches observed at top and bottom of casing is removed also low velocity patches at the right and left side casing is also removed.

Table 4 Achieved ICAC criteria

\begin{tabular}{|c|c|c|c|}
\hline AYERAGE YELOCITY (Y avg) & 0.85 & misec & \\
\hline 1.15 times $Y$ avg & 0.98 & misec & 0.7211 \\
\hline 1.40 times $\gamma$ avg & 1.19 & misec & 0.509 \\
\hline Total Yelocity Readings & 110 & Nos. & \\
\hline No. of readings vithin 1.15 times $Y$ avg & 94 & $\ln \%$ & $85 \%$ \\
\hline No. of readings vithin 1.40 times $Y$ avg & 90 & $\ln \%$ & $82 \%$ \\
\hline Standard Deviation & 0.19 & & \\
\hline RMS in \% & & $\ln \%$ & \\
\hline
\end{tabular}

Velocity reading at 110 number of points are taken at the end of first field to validate the data. It is observed that velocity readings at the end of first field follows ICAC criteria shown in fig.15. This data is validate with actual working electrostatic precipitator.

\section{Conclusion}

It is seen that by using non uniform porosity distribution screen flow through electrostatic precipitator distributed uniformly. By adjusting the porosity in the high velocity and low velocity region flow can be optimized. From table 4 it is seen that $85 \%$ number of readings are within 1.15 times $\mathrm{V}_{\text {avg. }}$ and 82 $\%$ number of readings are within 1.4 times $\mathrm{V}$ avg. it conforms the more uniformity in the flow and increases collection efficiency of the electrostatic precipitator.

\section{References}

B. Sahin and A. J. Ward-Smith, (1986), The use of perforated plates to control the flow emerging from a wide-angle diffuser, with application to electrostatic precipitator design, Heat and Fluid Flow, Vol 8, 124-131.

B.Y. Guoa, Q.F. Houa, A.B. Yua, L.F. Li b, J. Guob, (2012), Numerical modelling of the gas flow through perforated

Plates, chemical engineering research and design, 91 (2 $\left.\begin{array}{llll}2 & 0 & 1 & 3\end{array}\right)$ 403-408.

Dr. Basharat Salim, (2013), effect of geometrical parameters on the performance of wide angle diffusers, International Journal of Innovative Research in Science, Engineering and Technology, ISSN: 2319-8753, 7841-4191.

B. ahin, A.j. ward-smith, (1989), effect of perforated plates on wide-angle diffuser-exit velocity profiles, Journal af Wind Engineering and Industrial Aerodynamics, 34 (1990) 113 125.

S. Shaaban (2015) on the performance of perforated plate with

Optimized hole geometry, Flow Measurement and Instrumentation, JFMI1123.

Zheng Huang, Yan-Bin Xiong, (2014) Experimental investigation of full-coverage effusion cooling through perforated flat plates, Applied Thermal Engineering, 76 (2015) 76-85.

Shah M. E. Haque, M. G. Rasul, M. M. K. Khan, A. V. Deev, and N. Subaschandar, (2007) Numerical modelling for optimizing flow distribution inside an electrostatic precipitator, International journal of mathematics and computers in simulation, Issue 3, Volume 1. 